\title{
Response of the Cavity Shear Layer Oscillations to External Forcing
}

\author{
M. Gharib* \\ California Institute of Technology, Pasadena, California
}

\begin{abstract}
Influence of externally forced initial flow conditions on axisymmetric cavity shear layer was studied. A sinusoidally heated strip upstream of the cavity excited Tollmien-Schlichting waves that, after amplification by the boundary layer, were introduced to the cavity shear layer. It is shown that by selecting a forcing frequency, which satisfies a phase difference criterion between two corners of the cavity and has an amplitude that is above the threshold amplitude, it is possible to excite a naturally nonoscillating shear layer. It is also shown that the frequency and amplitude of the oscillation in the self sustained mode can be controlled through external forcing. By using a feed-back control scheme, up to $40 \%$ reduction of the velocity fluctuation level can be obtained.
\end{abstract}

\section{Nomenclature}

$A$

$A_{t h}=$ threshold amplitude

$A^{\text {th }} \quad$ = cavity width

$b_{\min }$

$d$

F

$H$

$N$

$s$

$U_{e}$

$U_{c}$

$\bar{u}$

$u^{\prime}$

$v^{\prime}$

$\frac{v}{u^{\prime} v}$

$u^{\prime} v$

$\frac{\bar{u} \bar{v}}{u^{\prime} v^{\prime}} / U_{e}^{2} I_{\max }$

$x$

$y$

$Y_{0.95}$

$\Delta_{0}$

$\theta_{0}$

$\phi$

$=$ minimum cavity width for the onset of the oscillation

$=$ model diameter

= shear layer oscillation frequency

$=$ boundary layer shape factor

$=$ integer number indicative of the oscillation mode

$=$ distance along the model nose

$=$ mean velocity at the separation point outside the boundary layer

$=$ phase velocity of the perturbation

$=$ mean streamwise velocity

$=$ streamwise velocity fluctuation

$=$ transverse velocity fluctuation

$=$ mean transverse velocity

$=$ Reynolds stress

$=$ mean nomentum transfer

$=$ maximum nondimensional Reynolds stress

$=$ streamwise distance

$=$ transverse distance

$=$ transverse location corresponding to $u / U_{e}=0.95$

= boundary layer displacement thickness at the separation point

$=$ boundary layer momentum thickness at the separation point

$=$ phase difference

\section{Introduction}

$S$ part of a study of the relation between the drag of a cavity and the processes occurring in its separated shear layer (Gharib ${ }^{1}$ ), a study was made of the effects of controlled forcing. This is a particularly useful configuration for such a study because, even without forcing, the oscillations in the shear layer tend to be coherent in phase due to the feedback

Presented as Paper 85-0528 at the AIAA Shear Flow Control Conference, Boulder, CO, March 12-14, 1985; received Sept. 17, 1985; revision received Feb. 24, 1986. Copyright (C) American Institute of Aeronautics and Astronautics, Inc., 1986. All rights reserved.

*Assistant Professor of Mechanical Engineering, Department of Applied Mechanics and Engineering Sciences. Member AIAA. condition generated by the impingement of the shear layer on the downstream corner of the cavity. The impingement process influences the initial region of the shear layer. The reception and amplification of this upstream influence by the initial sensitive region of the shear layer provides the necessary conditions for the cavity shear layer oscillations to be self sustained (Rockwell and Naudascher ${ }^{2}$ ). A minimum cavity width ( $b \mathrm{~min}$ ) is required for the initiation of self sustained oscillation. The onset of oscillation is associated with a decrease in frequency as the cavity width $(b)$ increases. This trend, then, is interrupted by a jump to a higher frequency and another similar reduction of frequency at a higher mode. Figure 1 presents a typical cartoon of the frequency variation with the cavity width. The self sustained oscillation and its associated mode switching phenomena eventually are replaced at a certain cavity width by a wake-like flow. In the present study, this flow regime will be referred to as "wake mode." Flow in the wake mode shows intermittent behavior on a much larger scale and is associated with high drag coefficient values for the cavity. Gharib and Roshko ${ }^{3}$ showed that the cavity drag is related directly to the state of the shear layer. The state of the shear layer is defined by the Reynolds stress $\left(\overline{u^{\prime} v^{\prime}}\right)$ and the mean momentum transfer $(\bar{u} \bar{v})$ in the shear layer.

This paper will discuss a series of experiments on the application of a new shear layer forcing scheme to manipulate the state of the cavity shear layer and, consequently, to control the cavity drag.

\section{Shear Layer Forcing Through Tollmien-Schlichting (T-S) Waves}

The main objectives of this study were to investigate the effect of the external perturbations, which were introduced to the initial sensitive region of the shear layer on the cavity flow. Therefore, a localized shear layer forcing technique as opposed to the global forcing techniques, ${ }^{4-6}$ where the whole shear layer experiences the forcing effects, was required.

A localized forcing of the shear layers can be obtained through T-S waves. The forcing of the T-S waves in boundary layers has been practiced by previous investigators. ${ }^{7,8}$ However, to our knowledge, T-S waves have not been used to force a separated shear layer resulting from such a boundary layer. In the present study, inspired by the experiment of Liepmann et al., ${ }^{8}$ periodic heating of a thin film was used to excite the T-S waves in the boundary layer upstream of the cavity shear layer, benefiting from amplification of the waves before their introduction into the shear layer (Fig. 2). One can imagine that the effect of the T-S waves or transverse velocity perturbation at the upstream corner of the cavity would be analogous to that of the oscillating flap at the leading edge of the splitter plate in Oster and Wygnanski's ${ }^{9}$ experiment. It will be seen 
later that the frequencies needed to excite Kelvin-Helmholtz waves in the free shear layer are available in the range of boundary layer $\mathrm{T}-\mathrm{S}$ waves.

\section{Experimental Set-Up}

The experiments were made in a water tunnel on a 4-in.-diam axisymmetric body with cavity as shown in Fig. 2. Velocity fluctuation measurements were made by a twocomponent laser doppler velocimeter, LVD (see Gharib ${ }^{1}$ or Gharib and Roshko ${ }^{3}$ for details of the model and the experimental set-up).

A single strip heater was flush mounted on the ellipsoidal nose of the model at $s=9.2 \mathrm{~cm}$, where $s$ is the distance on the model surface from the nose of the model. The strip heater was made of stainless steel ribbon $3.7 \mathrm{~mm}$ wide, and $0.51 \mathrm{~mm}$ thick. Figure 2 shows the location of the strip heater.

A function generator, in conjunction with a dual channel power amplifier, was used to supply current at selected frequency to the strip heater. Due to the quadratic dependence of Joule heating on the voltage, the input frequency to the power amplifier had to be half the desired forcing frequency.

\section{Operational Conditions}

The measurements were made at a fixed edge velocity $\left(U_{e}\right)$ of $22 \mathrm{~cm} / \mathrm{sec}$. The corresponding Reynolds number, based on the model diameter $d=10.16 \mathrm{~cm}$, was $24 \times 10^{3}$.

The boundary layer upstream of the cavity is laminar at $U_{e}=22 \mathrm{~cm} / \mathrm{sec}$ and has a shape factor of $H=\Delta_{0} / \Theta_{0}=2.5$, where $\Delta_{0}$ and $\theta_{0}$ are displacement and momentum thickness of the boundary layer at the upstream separation point. The Reynolds number based on $\theta_{0}$ was 95 .

The cavity flow response was measured in terms of the maximum Reynolds stress $\left(\overline{u^{\prime} v^{\prime}} /\left.U_{e}^{2}\right|_{\max }\right)$ that was developed in the shear layer at a station $0.254 \mathrm{~cm}\left(0.61 \theta_{0}\right)$ upstream of the downstream corner. Since the perturbations receive continuous amplification throughout the shear layer, the response of the cavity should be measured at the downstream corner. However, due to the finite size of the LDV's measuring volume, the closest point to the downstream corner that the LDV system could operate reliably was $0.254 \mathrm{~cm}$ upstream of the downstream corner.

We have convinced ourselves that the strip heater actually excites the T-S waves. This was done by comparing the cavity response generated by external perturbations from the strip heater installed at $s=9.2 \mathrm{~cm}$, to that of a second strip heater which was mounted at the upstream corner of the cavity. Even though the second heater was closer to the separation point, it was less efficient in inducing the oscillations in the cavity shear layer. For example, at a power level of $9 \mathrm{~W}$, the cavity showed no response to the second strip heater input while the same input power level by the first strip heater, generated strong response by the cavity shear layer. This observation indicates that similar to the T-S waves, the perturbations which are generated by the first heater receive continuous amplification through the boundary layer while benefitting from the adverse gradient associated with the shape of the model nose.

\section{Results and Discussion}

\section{External Forcing of the Nonoscillating Cavity Flow}

For small values of cavity width to depth ratio, $b / d$, for which the flow does not oscillate naturally, periodic oscillations were induced by forcing with the amplified T-S waves. The receptivity of the cavity shear layer in the nonoscillating regime to externally imposed disturbances was studied. Two different cavity widths were examined, with corresponding $b / \theta_{0}=66<\left(b / \theta_{0}\right)_{\min }$ and $b / \theta_{0}=77<\left(b / \theta_{0}\right)_{\min }$, where $\left(b / \theta_{0}\right)_{\min }$ is the minimum value for initation of the self sustained oscillations. Throughout this section, these selected widths will be referred to as case $A$ and $B$ respectively.

Spectral analysis of the response velocity fluctuations indicated a one to one relation between forcing and the response frequency. For case A, Fig. 3 presents the response of the cavity shear layer to a wide range of the forcing frequencies at different levels of forcing power. The results indicate that the amplitude level of shear layer response increases as the forcing power increases. For forcing levels above $9 \mathrm{~W}$, resonance peaks appear at the forcing frequency of $7.2 \mathrm{~Hz}$.

An overall phase measurement between two corners of the cavity showed that case $A$ at the resonance $(F=7.2 \mathrm{~Hz})$ satisfies the phase criterion $\phi / 2 \pi=F b / U_{c}=2$, where $\phi$ is the overall phase difference between two corners, $F$ is the response frequency, $b$ is the cavity width and $U_{c}$ is the phase velocity. In this study, each mode of the cavity shear layer oscillations has been identified by the integer value of the Strouhal number $\left(F b / U_{c}=N\right)$. Therefore, case $A$ at the resonance simulates the second mode of the oscillations. Removing the forcing caused the resonance mode to disappear, thus showing that the flow oscillations were not self sustaining. Figure 3 also shows that the existence of the resonance depends on a minimum amplitude threshold.

Figure 4 presents the response of the cavity shear layer for case $A$ and $B$ at a fixed forcing level of $0 \mathrm{~W}$. Case B shows a resonance peak at the forcing frequency of $6.2 \mathrm{~Hz}$ with

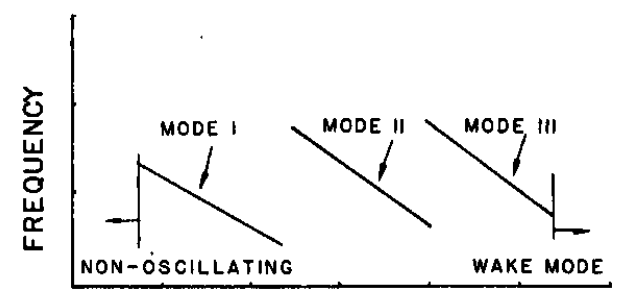

CAVITY WIDTH (b)

Fig. 1 Frequency variation of cavity shear layer with varying cavity width.

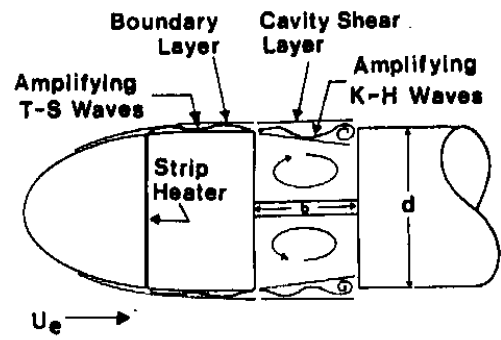

Fig. 2 Schematic of cavity model illustrating the strip heater technique.



Fig. 3 Variation of maximum Reynolds stress with forcing frequency at four different forcing power levels (case $A$ ). 


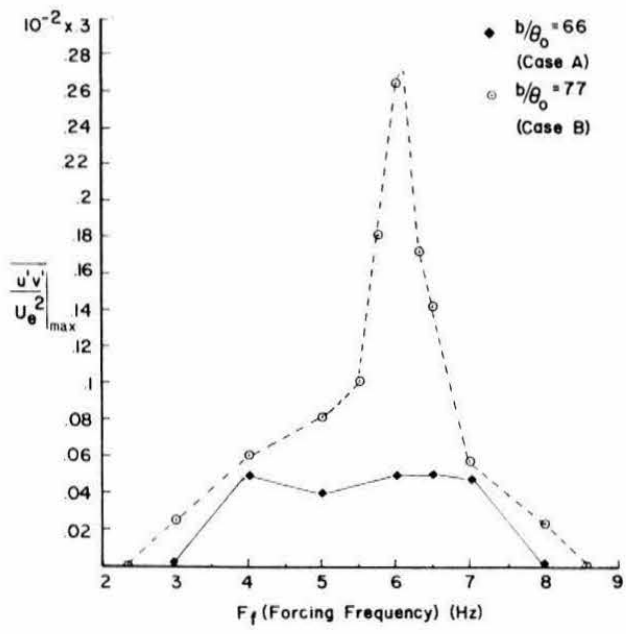

Fig. 4 Variation of the maximum Reynolds stress with forcing frequency, constant power.

$\phi / 2 \pi=F b / U_{c}=2$. Appearance of a resonance peak for case B at a lower forcing level than that of case $A$, indicates that the threshold level decreases as $b / \Theta_{0}$ increases. In case $\mathrm{B}$, the effect of initial lower amplitude of the forcing was compensated by longer cavity length in such a way that reduced the required forcing level for the resonance condition. The effect of higher forcing power on the exponential growth of the perturbations in the shear layer was to increase the general level of Reynolds stress (Fig. 5a). Figure 5b presents the growth behavior of the different frequencies for a fixed forcing level $(9 \mathrm{~W})$ for case $\mathrm{B}$.

It can be concluded that external frequencies can be imposed on naturally nonoscillating cavity flows, within the receptivity range of the shear layer. These frequencies can cause the cavity flow to resonate if they satisfy the following conditions: 1) $\phi / 2 \pi=F b / U_{c}=N$ for the given width $(b)$; and 2) $A>A_{t h}$ where $A$ is the amplitude of the external frequency at the downstream corner and $A_{t h}$ is the threshold amplitude.

It is logical to propose that, as the cavity width increases, the threshold level decreases to such an extent that a flow background frequency, which satisfies the phase criterion and has sufficient amplitude, will initiate the self sustained oscillation. In this study, the natural self sustained oscillations always started at the second mode. It is now clear that due to the insufficient cavity width and the absence of proper frequencies in the flow background, the first mode did not appear naturally. However, by selecting a proper forcing frequency and initial amplitude, the first mode of oscillations, $\phi / 2 \pi=F b / U_{c}=1$, was simulated for case A. This was accomplished at a forcing frequency of $4 \mathrm{~Hz}$, and a power level of $35 \mathrm{~W}$. A flow visualization of this case is shown in Fig. 6b. A comparison to the unforced case, shown in Fig. 6a, reveals two noteworthy features. The first is the appearance of a small amplitude wave in the shear layer of the forced case. The second is the interaction of this wave with the downstream corner and the resultant strong recirculating flow inside the cavity.

\section{Generating Drag Through Forcing}

The drag coefficient of the cavity was determined by integrating the mean and turbulent momentum transfer terms $\left(\hat{u} \bar{v}+\overline{u^{\prime} v^{\prime}}\right)$ along a straight line $(y=0)$ that connects two corners of the cavity (see Gharib and Roshko ${ }^{3}$ for details). Case $\mathrm{B}$, at resonance, had a drag coefficient of 0.0012 . This drag coefficient was one order of magnitude higher than that of the unforced case. Distributions of $\bar{u} \bar{v} / U_{e}^{2}$ and $\overline{u^{\prime} v^{\prime}} / U_{e}^{2}$ along the straight line that connects two corners, for the forced and unforced cavity, are given in Figs. 7a and $7 \mathrm{~b}$. These figures show that the difference in the drag coefficient of forced and unforced cases can be attributed to the development of a strong Reynolds stress level in the forced case.
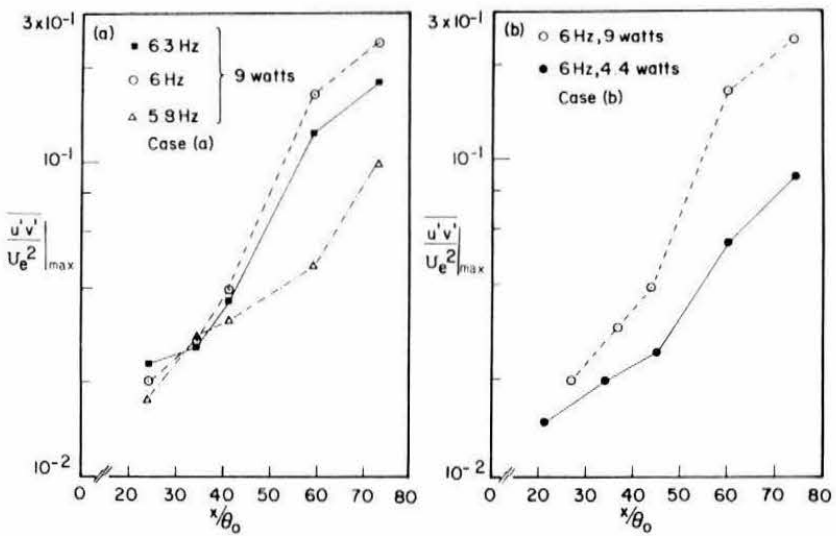

Fig. 5 Distribution of the maximum Reynolds stress with the streamwise distance: a) three different forcing frequencies, constant power, b) two different forcing powers, constant frequency.

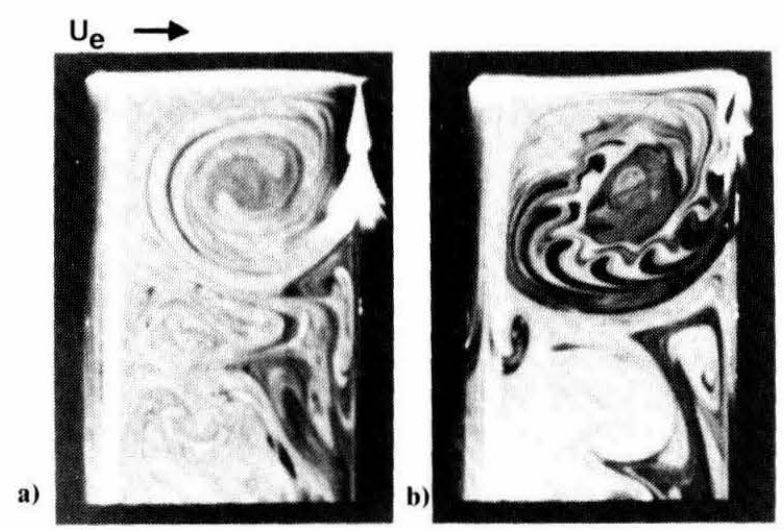

Fig. 6 Flow visualization of a naturally nonoscillating cavity flow, $b / \theta_{0}=66:$ a) unforced, b) forced.
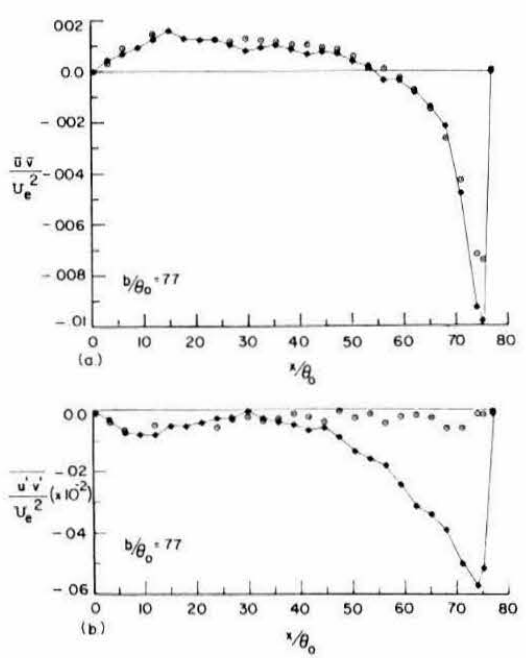

Fig. 7 Distribution of a) $\tilde{u} \bar{v} / u^{2}$ term, b) Reynolds stress, $\odot$ unforced, - forced.

\section{External Forcing Effects on a Naturally \\ Oscillating Cavity Flow}

Once the cavity flow had established the self sustained oscillations, $b / \theta_{0}>\left(b / \theta_{0}\right)_{\min }$, the flow resisted any external disturbances. Therefore, it was expected in order to interact with the flow oscillations, the amplitude level of external forcing must be comparable to that of the self sustained fluctuations in the flow.

A nondimensional cavity width of $b / \theta_{0}=95$ with an oscillation frequency of $5.4 \mathrm{~Hz}$ was selected for an external forcing 

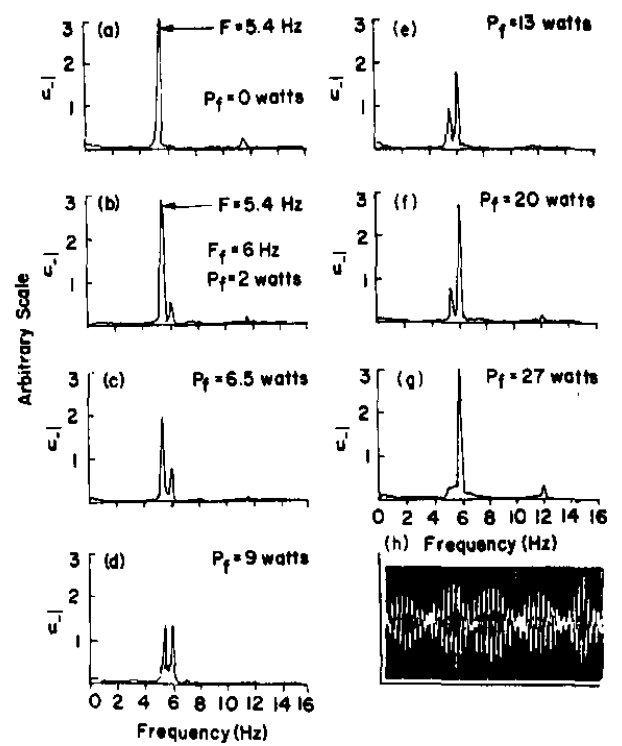

(h) Frequency $\{\mathrm{Hz}\}$

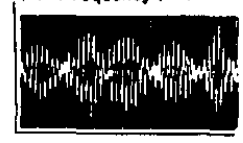

Frequency $(\mathrm{Hz})$

Fig. 8 Velocity fluctuation spectra of the natural and forced frequencies interaction, (h) scope trace of case (d).

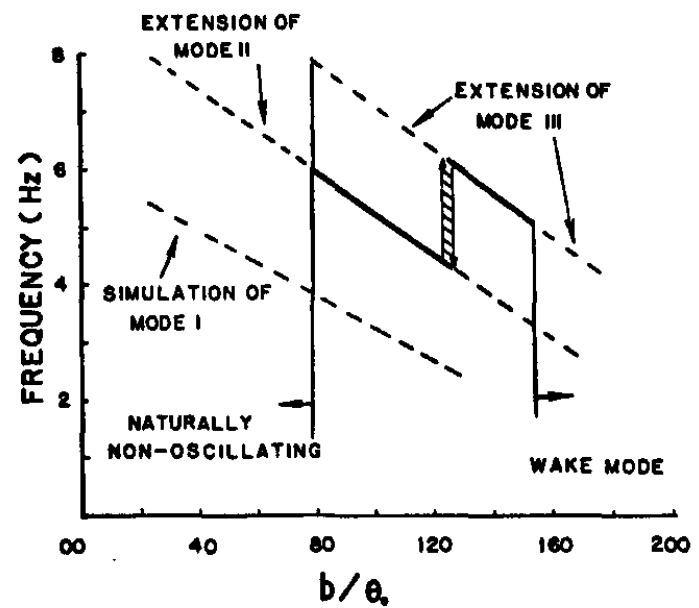

Fig. 9 Possible interactions with the cavity flow through external forcing: 1) extension of mode II and III, 2) simulation of mode I, and 3) delay of the wake mode.

experiment. An external forcing frequency of $6 \mathrm{~Hz}$ was introduced to the shear layer. Depicted in Figs. $8 \mathrm{a}-8 \mathrm{~g}$ are the spectral distributions of velocity fluctuations for several levels of forcing as the forcing power was changed from $2-27 \mathrm{~W}$.

The spectra, which were made at $x=60 \theta_{0}$ and $Y_{0.95}$ (the latter being the $y$ location of $\bar{u} / U_{e}=0.95$ ), show that, as the amplitude of the forcing increases, the amplitude of natural oscillation decreases and eventually disappears. At the forcing power of $27 \mathrm{~W}$, the forced frequency becomes the dominant frequency of the oscillations. A time trace of the signal for the case when both natural and forced frequency have the same amplitude, as seen in Fig. 8d, is given in Fig. 8h. A strong beat pattern in the signal indicates that both frequencies are present simultaneously. Note that in the coexistence region, Figs. $8 \mathrm{~b}-8 \mathrm{f}$, the peak amplitude of both frequencies is lower than that of the single frequency regions of Figs. 8a and $8 \mathrm{~g}$.

Miksad, ${ }^{10}$ in his study of dual excitation of a free shear layer, observed a similar interaction of two frequencies. He reported that if the excitation amplitudes were adjusted so that both disturbances reached a finite amplitude together, the final amplitudes of both components tended to be reduced, but not as strongly as when one component was clearly dominant. Miksad concluded that the distortion of the mean flow (a)

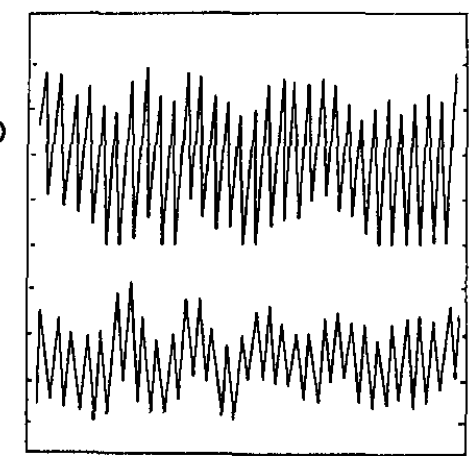

Fig. 10 Damping of natural oscillations: a) natural oscillation and b) strip heater on.

by the stronger frequency made the velocity field unsuitable for the growth of the weaker frequency.

Important to our study is the fact that the amplitude of the forced frequency in Fig. $8 \mathrm{~g}$, where natural frequency just disappears, is equal to the amplitude of the natural frequency in the absence of the forced frequency (Fig. 8a). This fact establishes the amplitude of the natural frequency as the level that should be exceeded by the amplitude of forced frequency to change the frequency of the oscillations. With this rule, it is possible to impose forced frequencies on the cavity shear layer within the receptivity range of the shear layer. These forced oscillations are not, in general, self sustained.

Switching of modes have been observed to be associated with hysteresis. Extension or termination of a desired mode could be achieved by providing a proper frequency within the hysteresis region. Therefore, the mode switching phenomena could be eliminated by external forcing. Also, transition to the wake mode could be prevented by enhancing the amplitude of the natural frequency through external forcing. It is possible to extend mode III, the last mode in this study, by 20 momentum thicknesses through external forcing. Drag measurements indicated a low cavity drag in the extended mode. This fact supports the conclusion of Gharib and Roshko ${ }^{3}$ that the self sustained oscillation is the main reason for the existence of the low-drag regimes of the cavity. However, once the cavity entered the wake mode, it was not possible to re-establish cavity flow oscillations by external forcing. A map of possible interaction with the cavity flow oscillations observed in this experiment is given in Fig. 9.

\section{Cancellation Experiment}

The cavity shear layer differs from a free shear layer by its imposed boundary condition at the downstream corner. Studies of Gharib and Roshko, ${ }^{3}$ as well as previous studies, indicate a strong phase coherence throughout the shear layer, which is considered to be the result of the downstream corner feedback through the shear layer. This phase stability is responsible for the appearance of a sharp peak in the power spectrum of velocity fluctuations. The velocity power spectrum of an unforced free shear layer does not have such a distinct, sharp peak, but rather a broad band around the natural frequency (Browand ${ }^{11}$ ).

The phase stability of a cavity controlled shear layer makes the possibility of active control of the oscillation quite appealing. This section describes an effort to reduce the amplitude of the oscillations by implementing the cancelling effect of two similar waves with finite phase difference. A cavity width of $b / \theta_{0}=82$, with a frequency of $6 \mathrm{~Hz}$, was selected for the cancellation experiment. The signal from a downstream location was fed to a function generator which had a built-in phase-lock loop. It was possible to lock to lower harmonics of the input signal. Phase variation of the output signal, with respect to the first subharmonic of the input signal, was possible through an independent manual adjustment knob. The 
power amplifier then was supplied by the output of the function generator.

Due to small drift in phase of the cavity flow oscillation, it was not possible to maintain a phase lock situation for an extended period of time. A correct phase angle occasionally could be found where the forced and natural frequencies would interact in such a way that wide band rms fluctuations were reduced. A sample of such a cancellation is shown in Fig. 10. A reduction of $\mathrm{rms}$ fluctuation by a factor of 2 was ahieved during 30 cycles of the cavity shear layer oscillations. This corresponds to a drag coefficient reduction by a factor of 10. Liepmann and Nosenchuck ${ }^{12}$ report a factor of 2 in the reduction of rms fluctuations of the natural (T-S) waves in the flat plate boundary layer.

\section{Concluding Remarks}

This study shows that control of the cavity flow oscillation, in the frequency and the amplitude domains, is possible by external forcing of the cavity shear layer. It is shown that excited Tollmien-Schlichting waves can be used to force the shear layer generated after separation of a boundary layer, that is, a transformation of the T-S waves of the boundary layer to Kelvin-Helmholtz waves of the shear layer is possible for some range of frequency.

Through the study of cavity layer response, it is found that minimum cavity length required for the onset of oscillation strongly depends on the turbulent content of the flow background. Different mode extension and simulation is possible by providing the proper forcing frequency and amplitude to the cavity shear layer. The most important result of the external forcing is the ability to control established self sustained oscillations. A $40 \%$ reduction in the amplitude of oscillations was obtained through the active interaction of external and natural perturbations.

\section{Acknowledgments}

This paper is based on work from the Ph.D. Thesis by the author, which was carried out at the Jet Propulsion Laboratory, California Institute of Technology. The work was sponsored by U. S. Army Research Office Contract 7134E. The author is grateful for assistance in various forms from A. Roshko, H. W. Liepmann, D. M. Nosenchuck, and V. Sarohia.

\section{References}

${ }^{1}$ Gharib, M., "The Effect of Flow Oscillations on Cavity Drag and a Technique for Their Control," Ph.D Thesis, California Institute of Technology, 1983.

${ }^{2}$ Rockwell, D. and Naudascher, E., "Review of Self Sustaining Oscillations of Flow Past Cavities," Transactions of ASME, Journal of Fluids Engineering, Vol. 100, 1978, pp. 152-165.

${ }^{3}$ Gharib, M. and Roshko, A., "The Effect of Flow Oscillations on Cavity Drag," Journal of Fluid Mechanics, to be published.

${ }^{4}$ Miksad, R. W., "Experiments on the Nonlinear Stages of Free Shear Layer Transition," Journal of Fluid Mechanics," Vol. 56, 1972 , p. 695.

${ }^{3}$ Ho, C. H. and Huang, L. S., "Sub-harmonics and Vortex Merging in Mixing Layers," Journal of Fluid Mechanics, Vol. 119, 1982, pp. 443-473.

${ }^{6}$ Roberts, F. A., "Effect of a Periodic Disturbance on Structure and Mixing in Turbulent Shear Layers and Wakes," Ph.D Thesis, California Institute of Technology, 1985.

${ }^{7}$ Schubauer, G. B, and Skramstad, H. K., "Laminar BoundaryLayer Oscillations and Stability of Laminar Flow," Journal of Aeronautical Science, Vol. 14, 1947, pp. 68-78.

${ }^{8}$ Liepmann, H. W., Brown, G. L., and Nosenchuck, D. M., "Control of Laminar-Instability Waves using a New Technique," Journal of Fluid Mechanics, Vol. 118, 1982, pp. 187-200.

${ }^{9}$ Oster, D. and Wygnanski, I., "The Forced Mixing Layer between Parallel Streams," Journal of Fluid Mechanics, Vol. 123, 1982, pp. 91-130.

${ }^{10}$ Miksad, R. W., "Experiments on Nonlinear Interactions in the Transition of a Free Shear Layer," Journal of Fluid Mechanics, Vol. 65,1973, p. 695

"Browand, F. K., "An Experimental Investigation of the Instabilities of an Incompressible, Separated Shear Layer," Journal of Fluid Mechanics, Vol. 26, 1966, pp. 281-307.

${ }^{12}$ Liepmann, H. W. and Nosenchuck, D. M., "Active Control of Laminar-Turbulent Transition," Journal of Fluid Mechanics, Vol. 118, 1982, pp. 201-204. 